\title{
Erratum to: Termini effects on the optical properties of graphene nanoribbons ${ }^{\star, \star \star}$
}

\section{Eur. Phys. J. B (2018) 91: 286, https://doi.org/10.1140/epjb/e2018-90179-4}

Claudia Cardoso $^{\mathrm{a}}$, Andrea Ferretti, and Deborah Prezzi ${ }^{\mathrm{b}}$

CNR-Nanoscience Institute, S3 Center, via G. Campi 213/a, 41125 Modena, Italy

Received 26 November 2018

Published online 17 December 2018

(C) EDP Sciences / Società Italiana di Fisica / Springer-Verlag GmbH Germany, part of Springer Nature, 2018

Figure 3 was not correct in the published article. This erratum provides the correct Figure 3:

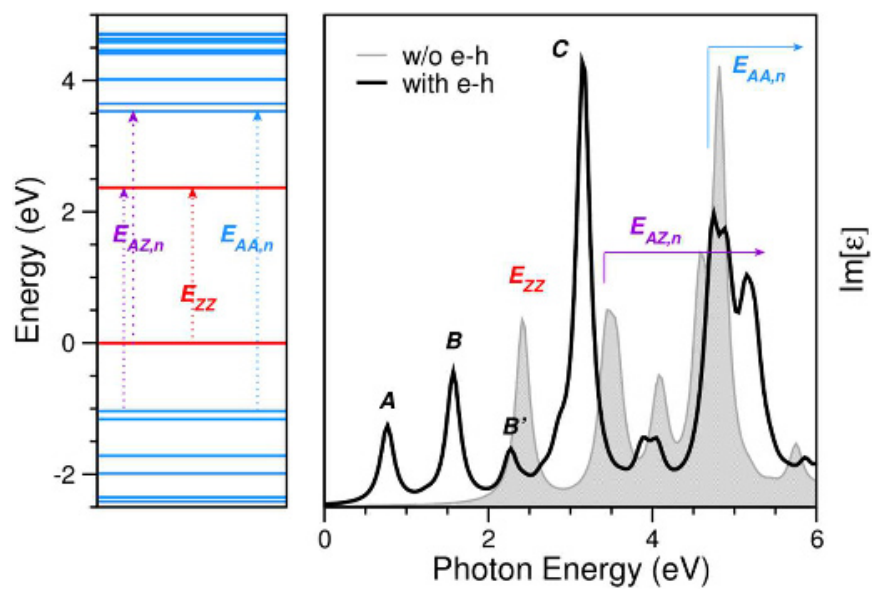

Fig. 3. Left: GW-corrected energy level scheme of $(7,10)$-AGNR. Tamm states are displayed in red, while bulk-like states are in blue. Right: Optical absorption spectrum of $(7,10)$-AGNR. The independent particle spectrum is in grey (shaded area), whereas the spectrum including e-h interaction at the BSE level is reported in black.

The original article has been corrected.

\footnotetext{
* Contribution to the Topical Issue "Special issue in honor of Hardy Gross", edited by C.A. Ullrich, F.M.S. Nogueira, A. Rubio, and M.A.L. Marques.

${ }^{\star \star}$ The online version of the original article can be found at https://doi.org/10.1140/epjb/e2018-90179-4

a Present address: International Iberian Nanotechnology Laboratory, Avenida Mestre José Veiga s/n 4715-330 Braga, Portugal.

b e-mail: deborah.prezzi@nano.cnr.it
} 